\title{
Spin Dynamics from Time-Dependent Spin-Density-Functional Theory
}

\author{
Zhixin Qian and Giovanni Vignale \\ Department of Physics, University of Missouri, Columbia, Missouri 65211
}

(Received 23 July 2001; published 17 January 2002)

\begin{abstract}
We derive the spin-wave dynamics of a magnetic material from the time-dependent spin-densityfunctional theory in the linear response regime. The equation of motion for the magnetization includes, besides the static spin stiffness, a "Berry curvature" correction and a damping term. A gradient expansion scheme based on the homogeneous spin-polarized electron gas is proposed for the latter two quantities, and the first few coefficients of the expansion are calculated to second order in the Coulomb interaction.
\end{abstract}

DOI: 10.1103/PhysRevLett.88.056404

The study of the ground-state properties of magnetic materials within the framework of spin-density-functional theory (SDFT) is by now a mature field [1-3]. By comparison, the study of excited-state properties is still in its infancy. There has been great interest in recent years in deriving a closed equation of motion for the magnetization starting from a first-principles description of the electrons as itinerant particles [4-8], rather than from the time-honored Heisenberg model of interacting local moments [9-11]. An alternative approach is to calculate the spectrum of spin excitations from the imaginary part of the linear spin-spin response function [12]. Our objective in this Letter is to unify the two approaches within the framework of the time-dependent SDFT. We emphasize new aspects of the physics beyond the adiabatic approximation (namely, dissipation), as well as a practically workable computational scheme.

Elementary spin excitations in itinerant-electron magnets fall into two groups (i) Stoner excitations - in which a single electron quasiparticle is spin reversed and (ii) spin waves. Both types of excitations can be computed from the linear spin-spin response function $\chi_{i j}\left(\mathbf{r}, \mathbf{r}^{\prime} ; \omega\right)=i \beta^{2} \int_{0}^{\infty} d t e^{i \omega t}\left\langle\left[\hat{S}_{i}(\mathbf{r}, t), \hat{S}_{j}\left(\mathbf{r}^{\prime}\right)\right]\right\rangle\left[\hat{S}_{i}(\mathbf{r})\right.$ is the $i$ component of the spin-density operator, with $\beta=$ $g e /(2 m c)$ and $\hbar=1]$, which determines the magnetization $m_{i}(\mathbf{r}, \omega)=-\beta\left\langle S_{i}(\mathbf{r}, \omega)\right\rangle$ induced by an external magnetic field $\mathbf{B}\left(\mathbf{r}^{\prime}, \omega\right)$ at a frequency $\omega$ :

$$
m_{i}(\mathbf{r}, \omega)=\sum_{j} \int \chi_{i j}\left(\mathbf{r}, \mathbf{r}^{\prime} ; \omega\right) B_{j}\left(\mathbf{r}^{\prime} ; \omega\right) d \mathbf{r}^{\prime} .
$$

Stoner excitations are distributed along branch cuts of this response function, while collective modes show up as isolated poles in the complex frequency plane.

A time-dependent SDFT is ideally suited for calculating $\chi_{i j}[13]$. As a first step in this direction one solves the static Kohn-Sham equation [14], whose eigenfunctions and eigenvalues determine the exact equilibrium density and magnetization. One then constructs the linear spin-spin response function $\chi_{\mathrm{KS}, i j}\left(\mathbf{r}, \mathbf{r}^{\prime} ; \omega\right)$ of the Kohn-Sham (KS) system. Because this is a stationary noninteracting system, the calculation can be carried out exactly [15]. Finally, the response of the physical system is calculated as the response of the KS system to an effective time-
PACS numbers: 71.15.Mb, 75.30.Ds, 76.50. $+\mathrm{g}$

dependent field $\mathbf{B}_{\text {eff }}$ which includes, in addition to the external field B, a many-body "exchange-correlation" field $\mathbf{B}_{x c}$. When these ideas are cast in formulas one obtains the well-known mathematical relation between the matrix inverses of the exact response function and the KS response function, namely,

$$
\left[\chi^{-1}\right]_{i j}\left(\mathbf{r}, \mathbf{r}^{\prime} ; \omega\right)=\left[\chi_{\mathrm{KS}}^{-1}\right]_{i j}\left(\mathbf{r}, \mathbf{r}^{\prime} ; \omega\right)-f_{x c, i j}\left(\mathbf{r}, \mathbf{r}^{\prime} ; \omega\right),
$$

where $f_{x c, i j}\left(\mathbf{r}, \mathbf{r}^{\prime} ; \omega\right)$ is the tensor that connects $\mathbf{B}_{x c}$ to the induced magnetization

$$
B_{x c, i}(\mathbf{r}, \omega)=\sum_{j} \int f_{x c, i j}\left(\mathbf{r}, \mathbf{r}^{\prime} ; \omega\right) m_{j}\left(\mathbf{r}^{\prime} ; \omega\right) d \mathbf{r}^{\prime} .
$$

Collective spin excitations can then be obtained from the solution of the eigenvalue problem

$$
\sum_{j} \int\left[\chi^{-1}\right]_{i j}\left(\mathbf{r}, \mathbf{r}^{\prime} ; \omega\right) m_{j}\left(\mathbf{r}^{\prime}, \omega\right) d \mathbf{r}^{\prime}=0,
$$

where $m_{j}(\mathbf{r}, \omega)$ is the magnetization profile in the spin wave. The problem is to find the frequencies $\omega$ for which this equation has nonvanishing solutions. Because $\chi$ has both real and imaginary parts these eigenfrequencies will be complex in general and will determine both the dispersion $(\operatorname{Re} \omega)$ and the linewidth $(\operatorname{Im} \omega)$ of spin waves.

To appreciate the power of Eqs. (2) and (4) we now use them to derive both the adiabatic spin dynamics [4-6] and the Landau-Lifshitz (LL) equation [16] in the linear response regime. First of all, we choose to focus on the transverse part of the response function, namely, the part that describes the response to a magnetic field perpendicular to the ground-state magnetization. It turns out that in a collinear magnet the transverse response function is rigorously decoupled from the longitudinal one in the absence of spin-orbit interactions. In general, this decoupling is justified by the difference between the time scales of the longitudinal and transverse spin dynamics. Our key assumption is that both $\chi_{\mathrm{KS}}^{-1}$ and $f_{x c}$ can be Taylor expanded, at low frequency, in powers of $\omega$. Keeping only the first order term in the low-frequency expansion of $\chi^{-1}$ we come to

$$
\left[\chi^{-1}\right]_{i j}\left(\mathbf{r}, \mathbf{r}^{\prime} ; \omega\right)=\alpha_{i j}\left(\mathbf{r}, \mathbf{r}^{\prime}\right)+i \omega \tilde{\Omega}_{i j}\left(\mathbf{r}, \mathbf{r}^{\prime}\right),
$$


where $\alpha_{i j}\left(\mathbf{r}, \mathbf{r}^{\prime}\right) \equiv\left[\chi^{-1}\right]_{i j}\left(\mathbf{r}, \mathbf{r}^{\prime} ; \omega=0\right)$ is the (symmetric) spin stiffness tensor, given by the second derivative of the ground-state energy with respect to the magnetization, and

$$
\tilde{\Omega}_{i j}\left(\mathbf{r}, \mathbf{r}^{\prime}\right) \equiv \lim _{\omega \rightarrow 0} \frac{\partial \operatorname{Im}\left[\chi^{-1}\right]_{i j}\left(\mathbf{r}, \mathbf{r}^{\prime} ; \omega\right)}{\partial \omega},
$$

with the derivative taken along the real frequency axis. $\left(\tilde{\Omega}_{i j}\right.$ is purely real because the derivative of $\operatorname{Re} \chi$ vanishes at $\omega=0$.) Notice that, by definition,

$$
\int \alpha_{i j}\left(\mathbf{r}, \mathbf{r}^{\prime}\right) m_{j}\left(\mathbf{r}^{\prime}\right) d \mathbf{r}^{\prime}=\frac{\delta E[\mathbf{m}]}{\delta m_{i}(\mathbf{r})},
$$

where $E[\mathbf{m}]$ is the ground-state energy regarded as a functional of $\mathbf{m}(\mathbf{r})$ [17]. The tensor $\tilde{\Omega}_{i j}$ can be split into antisymmetric and symmetric components as follows:

$$
\tilde{\Omega}_{i j}\left(\mathbf{r}, \mathbf{r}^{\prime}\right)=\Omega_{i j}\left(\mathbf{r}, \mathbf{r}^{\prime}\right)+\gamma_{i j}\left(\mathbf{r}, \mathbf{r}^{\prime}\right),
$$

where $\Omega_{i j}\left(\mathbf{r}, \mathbf{r}^{\prime}\right)=-\Omega_{j i}\left(\mathbf{r}^{\prime}, \mathbf{r}\right)$ and $\gamma_{i j}\left(\mathbf{r}, \mathbf{r}^{\prime}\right)=\gamma_{j i}\left(\mathbf{r}^{\prime}, \mathbf{r}\right)$. Therefore, substituting (5) into (4), and switching to a real time representation with the substitution $-i \omega \rightarrow \partial / \partial t$, we obtain the equation of motion

$$
\begin{aligned}
\sum_{j} \int d \mathbf{r}^{\prime}\left[\Omega_{i j}\left(\mathbf{r}, \mathbf{r}^{\prime}\right)\right. & \left.+\gamma_{i j}\left(\mathbf{r}, \mathbf{r}^{\prime}\right)\right] \frac{\partial m_{j}\left(\mathbf{r}^{\prime}, t\right)}{\partial t} \\
& =\frac{\delta E[\mathbf{m}]}{\delta m_{i}(\mathbf{r}, t)} .
\end{aligned}
$$

This equation reduces to the Niu-Kleinman adiabatic equation of motion [4] if the symmetric tensor $\gamma_{i j}$ is neglected and the antisymmetric tensor $\Omega_{i j}$ is identified with the "Berry curvature." Indeed, after a lengthy but straightforward calculation we can show that

$$
\Omega_{i j}\left(\mathbf{r}, \mathbf{r}^{\prime}\right)=-2 \operatorname{Im}\left\langle\frac{\partial \psi[\mathbf{m}]}{\partial m_{i}(\mathbf{r})} \mid \frac{\partial \psi[\mathbf{m}]}{\partial m_{j}\left(\mathbf{r}^{\prime}\right)}\right\rangle,
$$

where $\psi[\mathbf{m}]$ is the ground-state wave function regarded as a functional of $\mathbf{m}[17,18]$. Thus, the antisymmetric part of Eq. (6) is equivalent to Eq. (10): the former is, however, more amenable to approximation and computation.

The symmetric part of Eq. (6), $\gamma_{i j}$, is responsible for dissipation as one can immediately verify by calculating the rate of entropy production at temperature $T$ :

$$
\begin{aligned}
T \frac{d S}{d t} & =-\int \frac{\delta E[\mathbf{m}]}{\delta \mathbf{m}(\mathbf{r}, t)} \cdot \frac{\partial \mathbf{m}(\mathbf{r}, t)}{\partial t} d \mathbf{r} \\
& =-\sum_{i j} \int d \mathbf{r} \int d \mathbf{r}^{\prime} \frac{\partial \mathbf{m}_{i}(\mathbf{r}, t)}{\partial t} \gamma_{i j}\left(\mathbf{r}, \mathbf{r}^{\prime}\right) \frac{\partial \mathbf{m}_{j}\left(\mathbf{r}^{\prime}, t\right)}{\partial t} .
\end{aligned}
$$

Not surprisingly, this term is absent in a purely adiabatic theory such as that of Ref. [4].

We now turn to the task of approximating the righthand side of Eq. (6). A classic approximation scheme is provided by the gradient expansion [19]. In this scheme one assumes that the two-point function $\tilde{\Omega}_{i j}\left(\mathbf{r}, \mathbf{r}^{\prime}\right)$ is a short ranged function of the distance $\left|\mathbf{r}-\mathbf{r}^{\prime}\right|$. It is then permissible, if the density and magnetization are slowly varying, to expand $\tilde{\Omega}$ as

$$
\begin{aligned}
\tilde{\Omega}_{i j}\left(\mathbf{r}, \mathbf{r}^{\prime}\right)= & \tilde{\Omega}_{0, i j}[n(\mathbf{r}), m(\mathbf{r})] \delta\left(\mathbf{r}-\mathbf{r}^{\prime}\right) \\
& +\tilde{\Omega}_{2, i j}[n(\mathbf{r}), m(\mathbf{r})] \nabla_{\mathbf{r}} \delta\left(\mathbf{r}-\mathbf{r}^{\prime}\right) \cdot \nabla_{\mathbf{r}^{\prime}}+\ldots,
\end{aligned}
$$

where $\tilde{\Omega}_{0, i j}[n, m]$ and $\tilde{\Omega}_{2, i j}[n, m]$ are the coefficients of $q^{0}$ and $q^{2}$, respectively, in the small- $q$ expansion of $\tilde{\Omega}_{i j}^{\text {hom }}(\mathbf{q}) \equiv \int \tilde{\Omega}_{i j}^{\text {hom }}\left(\mathbf{r}-\mathbf{r}^{\prime}\right) e^{-i \mathbf{q} \cdot\left(\mathbf{r}-\mathbf{r}^{\prime}\right)} d \mathbf{r}$ in a homogeneous electron gas of density $n$ and magnetization $\mathbf{m}$.

We are now in a position to prove that the standard LL equation [16] is simply the zero-order approximation (i.e., the local density approximation) in the gradient expansion for the Berry curvature. To this end, we consider a homogeneous spin-polarized electron gas with the same ground-state density $n$ and magnetization $\mathbf{m}_{0}$ as those of the real system at point $\mathbf{r}$. The homogeneous magnetization is maintained by an external fictitious magnetic field $\mathbf{B}_{0}$ parallel to $\mathbf{m}_{0}$,

$$
B_{0}=\left(\frac{\partial \epsilon(n, m)}{\partial m}\right)_{m=m_{0}},
$$

where $\epsilon(n, m)$ is the energy density of the homogeneous electron gas of density $n$ and magnetization $m$ [20]. The transverse spin-spin response function of this system at $q=0$ is

$$
\left[\chi^{-1}\right]_{i j}(q=0, \omega)=\frac{\omega_{0} \delta_{i j}+i \omega \epsilon_{i j}}{\beta m_{0}},
$$

where $\omega_{0}=\beta B_{0}$ and $\epsilon_{i j}$ is the two-dimensional LeviCivita tensor with the Cartesian indices $i, j$ being orthogonal to the direction of $\mathbf{m}_{0}$. Thus, for the homogeneous electron gas, $\tilde{\Omega}_{i j}(q=0)=\epsilon_{i j} / \beta m_{0}$ [see Eq. (6)], and then, from Eqs. (8) and (12), we see that the local density approximation takes the form

$$
\Omega_{i j}\left(\mathbf{r}, \mathbf{r}^{\prime}\right) \simeq \epsilon_{i j} \frac{1}{\beta m_{0}(\mathbf{r})} \delta\left(\mathbf{r}-\mathbf{r}^{\prime}\right) .
$$

With this approximation Eq. (9) reduces to the linearized LL equation,

$$
\frac{\partial m_{i}(\mathbf{r}, t)}{\partial t}=-\sum_{j} \beta m_{0} \epsilon_{i j} \frac{\partial E[\mathbf{m}]}{\partial m_{j}(\mathbf{r})} .
$$

Notice that the dissipative $\gamma_{i j}$ is exactly zero at this order of approximation. Thus, the gradient expansion for $\gamma_{i j}$ begins with a second-order term

$$
\gamma_{i j}\left(\mathbf{r}, \mathbf{r}^{\prime}\right)=\gamma_{2, i j}(\mathbf{r}) \nabla_{\mathbf{r}} \delta\left(\mathbf{r}-\mathbf{r}^{\prime}\right) \cdot \nabla_{\mathbf{r}^{\prime}}+\ldots
$$

This makes physical sense because a global rotation of the spins must be rigorously undamped in the absence of spin-orbit interactions.

The remainder of this Letter is devoted to the calculation of the leading gradient corrections to the LL equation. After lengthy calculations, which will be described in 
detail elsewhere, we obtain the small- $q$ expansion of the transverse spin-spin response function of the homogeneous spin-polarized electron gas:

$$
\begin{aligned}
{\left[\chi^{-1}\right]_{i j}(\mathbf{q}, \omega)=\left[\chi^{-1}\right]_{i j}(0, \omega)+\frac{n q^{2}}{4 m m_{0}^{2}} \delta_{i j}+\frac{q^{2}}{\left(2 m m_{0}\right)^{2}} } \\
\times\left[\frac{4 m\left\langle\hat{T}_{\downarrow}-\hat{T}_{\uparrow}\right\rangle}{3 V} \frac{\omega_{0} \delta_{i j}-i \omega \epsilon_{i j}}{\omega^{2}-\omega_{0}^{2}}+\frac{2 \pi n^{2}[2 g(0)-1]}{3 m a_{0}}\left(\frac{\delta_{i j}-i \epsilon_{i j}}{\left(\omega-\omega_{0}\right)^{2}}+\frac{\delta_{i j}+i \epsilon_{i j}}{\left(\omega+\omega_{0}\right)^{2}}\right)\right. \\
\left.\quad+\frac{F_{-+}(\omega)\left(\delta_{i j}-i \epsilon_{i j}\right)}{\left(\omega-\omega_{0}\right)^{2}}+\frac{F_{+-}(\omega)\left(\delta_{i j}+i \epsilon_{i j}\right)}{\left(\omega+\omega_{0}\right)^{2}}\right],
\end{aligned}
$$

where $V$ is the volume and $a_{0}$ is the Bohr radius, $\hat{T}_{\uparrow}$ and $\hat{T}_{\downarrow}$ are the kinetic energy operators associated with up-spin and down-spin electrons, respectively, the angular brackets denote the ground-state or thermal ensemble average, $g(0)$ is the pair correlation function at zero separation, and $F_{+-}(\omega)$ is a four-point response function, defined as

$$
\begin{aligned}
F_{+-}(\omega)= & F_{-+}^{*}(-\omega) \\
= & \frac{1}{3 V^{3}} \sum_{\mathbf{k}, \mathbf{k}^{\prime}} v(\mathbf{k}) v\left(\mathbf{k}^{\prime}\right) \mathbf{k} \cdot \mathbf{k}^{\prime} \\
& \times\left\langle\left\langle\hat{S}_{+}(-\mathbf{k}) \hat{\rho}(\mathbf{k}) ; \hat{S}_{-}\left(\mathbf{k}^{\prime}\right) \hat{\rho}\left(-\mathbf{k}^{\prime}\right)\right\rangle\right\rangle_{\omega} .
\end{aligned}
$$

$\hat{S}_{ \pm}(\mathbf{k})=\hat{S}_{x}(\mathbf{k}) \pm i \hat{S}_{y}(\mathbf{k})$ are spin-density fluctuation operators, $v(\mathbf{k})=4 \pi e^{2} / k^{2}$, and $\hat{\rho}(\mathbf{k})$ is the density fluctuation operator. The "Zubarev product" is defined as $\langle\langle\hat{A} ; \hat{B}\rangle\rangle_{\omega} \equiv-i \int_{0}^{\infty} d t e^{i \omega t}\langle[\hat{A}(t), \hat{B}]\rangle$. Taking the small $\omega$ limit of this expression we obtain the coefficients of the gradient expansion for $\Omega$ and $\gamma$ as follows:

$$
\begin{aligned}
\Omega_{2, i j}= & \epsilon_{i j} \frac{1}{\left(m m_{0} \omega_{0}\right)^{2}}\left[\frac{m\left\langle T_{\uparrow}-T_{\downarrow}\right\rangle}{3 V}+\frac{2 \pi n^{2}[2 g(0)-1]}{3 m a_{0} \omega_{0}}\right. \\
& \left.+\frac{\operatorname{Re} F_{+-}(0)}{\omega_{0}}-\left.\frac{1}{2} \frac{\partial \operatorname{Re} F_{+-}(\omega)}{\partial \omega}\right|_{\omega=0}\right]
\end{aligned}
$$

and

$$
\gamma_{2, i j}=-\frac{\delta_{i j}}{2\left(m m_{0} \omega_{0}\right)^{2}} \lim _{\omega \rightarrow 0} \frac{\operatorname{Im} F_{+-}(\omega)}{\omega} .
$$

Since the long wavelength spin-wave frequency in a ferromagnet is proportional to $q^{2}$, the above results indicate that the gradient corrections to $\Omega$ and $\gamma$ will affect the dispersion and damping of ferromagnetic spin waves beginning at order $q^{4}$.

Equations (20) and (21) contain both ground-state (thermal ensemble) averages, such as $\left\langle T_{\uparrow}-T_{\downarrow}\right\rangle$ and $g(0)$, and the dynamical response function $F_{+-}(\omega)$, which depends on the spectrum of excited states. The former can be calculated to a high degree of accuracy by variational and diffusion Monte Carlo techniques [21]; the major challenge lies therefore in the calculation of $F_{+-}(\omega)$. The form of Eq. (19) suggests that we evaluate $F_{+-}(\omega)$ to second order in the Coulomb interaction: this is accomplished by substituting the noninteracting expression for the four point response function $\left\langle\left\langle\hat{S}_{+}(-\mathbf{k}) \hat{\rho}(\mathbf{k}) ; \hat{S}_{-}\left(\mathbf{k}^{\prime}\right) \hat{\rho}\left(-\mathbf{k}^{\prime}\right)\right\rangle\right\rangle_{\omega}$. Even such an approximate calculation turns out to be very difficult, but we have been able to establish analytically the limiting forms of the imaginary part of $F_{+-}(\omega)$ for high and low frequency. At low frequency $\left(\omega \ll E_{F \uparrow}-E_{F \downarrow}\right.$, where $E_{F \uparrow}$ is the larger of the two Fermi energies) we find

$$
\operatorname{Im} F_{+-}(\omega)=-\frac{m^{2} \Gamma \omega\left(\omega^{2}+4 \pi^{2} k_{B}^{2} T^{2}\right)}{36 \pi^{3} k_{F \uparrow} a_{0}^{2}},
$$

where $k_{B}$ is the Boltzmann constant and $T$ is the temperature. The dimensionless coefficient $\Gamma$ is the sum of "direct" and "exchange" terms: $\Gamma=\Gamma^{(D)}+\Gamma^{(E)}$ which we report separately for reasons that will become clear in the following:

$$
\begin{aligned}
\Gamma^{(D)} & =\frac{2 \lambda}{1-\lambda^{2}}+\frac{\theta(3 \lambda-1)(3 \lambda-1)}{2 \lambda(1-\lambda)}, \\
\Gamma^{(E)} & =\frac{1}{2} \ln \frac{1+\lambda}{1-\lambda}-\frac{\theta(3 \lambda-1)}{2 \lambda} \ln \frac{2 \lambda}{1-\lambda},
\end{aligned}
$$

where $\lambda \equiv(1-\zeta)^{1 / 3} /(1+\zeta)^{1 / 3}$, and $\zeta \equiv\left(n_{\uparrow}-n_{\downarrow}\right) / n$ is the degree of spin polarization, and $\theta$ is the step function. Equation (22) yields the damping tensor according to Eq. (21):

$$
\gamma_{2, i j}=\delta_{i j} \frac{\Gamma}{2 \pi k_{F \uparrow}\left(3 m_{0} \omega_{0} a_{0}\right)^{2}}\left(k_{B} T\right)^{2} .
$$

Note that the dissipation vanishes as $T^{2}$ for $T \rightarrow 0$ [22].

To calculate the correction to the Berry curvature we also need the real part of $F_{+-}(\omega)$. To this end, we make use of the Kramers-Krönig dispersion relation

$$
\operatorname{Re} F_{+-}(\omega)=\frac{1}{\pi} \int_{-\infty}^{\infty} d \omega^{\prime} \frac{\operatorname{Im} F_{+-}\left(\omega^{\prime}\right)}{\omega^{\prime}-\omega}
$$

and use for $\operatorname{Im} F_{+-}(\omega)$ at finite frequency the modedecoupling approximation of Ref. [23]:

$$
\begin{aligned}
\frac{1}{V^{2}} \operatorname{Im} & \left\langle\left\langle S_{+}(-\mathbf{k}) \rho(\mathbf{k}) ; S_{-}\left(\mathbf{k}^{\prime}\right) \rho\left(-\mathbf{k}^{\prime}\right)\right\rangle\right\rangle_{\omega} \\
\simeq & -g_{x} \frac{\delta_{\mathbf{k}, \mathbf{k}^{\prime}}}{\pi} \int_{0}^{\omega} \operatorname{Im} \chi_{n n}\left(\mathbf{k}, \omega-\omega^{\prime}\right) \\
& \times \operatorname{Im} \chi_{+-}\left(-\mathbf{k}, \omega^{\prime}\right) d \omega^{\prime},
\end{aligned}
$$

where $\chi_{n n}(\mathbf{k}, \omega)=V^{-1}\langle\langle\rho(\mathbf{k}) ; \rho(-\mathbf{k})\rangle\rangle_{\omega}$ is the densitydensity response function, and the factor $g_{x}=\left(\Gamma^{(D)}+\right.$ $\left.\Gamma^{(E)}\right) / \Gamma^{(D)}$ is used to include the exchange contribution and assure the correct behavior in the most important lowfrequency limit.

Carrying out the calculations, we find that the last two terms on the right-hand side of Eq. (20) are 


$$
\operatorname{Re} F_{+-}(0)=-g_{x} \frac{k_{F \uparrow}{ }^{3} E_{F \uparrow}}{3 \pi^{4} a_{0}^{2}} P(\zeta),
$$

and

$$
\left.\frac{\partial \operatorname{Re} F_{+-}(\omega)}{\partial \omega}\right|_{\omega=0}=-g_{x} \frac{k_{F \uparrow}^{3}}{3 \pi^{4} a_{0}^{2}} Q(\zeta) .
$$

$P(\zeta)$ and $Q(\zeta)$ can be very accurately parametrized as $P(\zeta)=1.9606-3.5 \zeta-1.4 \zeta^{2} \ln \zeta+2.08 \zeta^{2}$, and $Q(\zeta)=1.18 \zeta-0.186 \zeta^{2}-0.842 \zeta^{3}-(0.045 \zeta-$ $\left.1.49 \zeta^{2}\right) \ln \zeta$.

Finally, we need practical approximate expressions for the ground-state averages appearing in Eq. (20). The zero-separation pair-correlation function $g(0)$ of a spinpolarized electron gas has recently been calculated by Gori-Giorgi and Perdew, and an explicit expression is given in Eqs. (47) and (22)-(30) of their paper [24].

As for $\left\langle T_{\uparrow}-T_{\downarrow}\right\rangle$, in the absence of detailed calculations, we propose a linear interpolation for the correlation contribution:

$$
\left\langle T_{\uparrow}-T_{\downarrow}\right\rangle=\left\langle T_{\uparrow}-T_{\downarrow}\right\rangle_{0}-N \frac{d\left(r_{s} \epsilon_{c}\right)}{d r_{s}}(\zeta=1) \zeta,
$$

where $\epsilon_{c}$ is the correlation energy per particle, $r_{s}$ is the Wigner radius, and $\left\langle T_{\uparrow}-T_{\downarrow}\right\rangle_{0}=\left(k_{F \uparrow}{ }^{5}-k_{F \downarrow}{ }^{5}\right) / 20 \pi^{2} m$ is the difference of kinetic energies of the noninteracting systems.

The spin dynamics equation (9) is the central result of this Letter. It includes both the adiabatic spin dynamics and the conventional Landau-Lifshitz equation as special cases. It reduces to the adiabatic spin dynamics if the damping tensor $\gamma$ is neglected. It further reduces to a conventional Landau-Lifshitz equation if the gradient corrections to $\Omega$ are neglected. In addition, we have developed a density-functional scheme for the systematic calculation of $\Omega$ and $\gamma$. The analytical expressions for the damping tensor and the Berry curvature make the application of the new equation of motion to spin dynamics of ferromagnetic and antiferromagnetic systems quite promising. If greater accuracy is required, one can revert to the full-fledged linear response formalism, in which $\chi_{\mathrm{KS}}^{-1}$ is treated exactly and only $f_{x c}$ is approximated.

We gratefully acknowledge support from NSF Grant No. DMR-0074959 and Research Board Grant No. URB-00-029 at the University of Missouri. We thank John Perdew for his comments on the manuscript.

[1] U. von Barth and L. Hedin, J. Phys. C 5, 1629 (1972).
[2] A. K. Rajagopal, Adv. Chem. Phys. 41, 59 (1980), and references therein.

[3] L. M. Sandratskii, Adv. Phys. 47, 91 (1998); J. Sticht, K.-H. Höck, and J. Kübler, J. Phys. Condens. Matter 1, 8155 (1989).

[4] Q. Niu and L. Kleinman, Phys. Rev. Lett. 80, 2205 (1998).

[5] Q. Niu, X. Wang, L. Kleinman, W. M. Liu, D. M. C. Nicholson, and G. M. Stocks, Phys. Rev. Lett. 83, 207 (1999).

[6] R. Gebauer and S. Baroni, Phys. Rev. B 61, R6459 (2000).

[7] V.P. Antropov, M.I. Katsnelson, B. N. Harmon, M. van Schilfgaarde, and D. Kusnezov, Phys. Rev. B 54, 1019 (1996).

[8] D. M. Bylander, Q. Niu, and L. Kleinman, Phys. Rev. B 61, R11 875 (2000).

[9] C. Herring, in Magnetism, edited by G. T. Rado and H. Suhl (Academic Press, New York, 1966), Vol. IV.

[10] T. Moriya, Spin Fluctuations in Itinerant Electron Magnetism, Springer Series in Solid State Sciences Vol. 56 (Springer-Verlag, Berlin, 1985).

[11] A. Aharoni, Introduction to the Theory of Ferromagnetism (Oxford University Press, New York, 1996).

[12] S. Y. Savrasov, Phys. Rev. Lett. 81, 2570 (1998).

[13] For an introduction to time-dependent DFT, see E. K. U. Gross, J.F. Dobson, and M. Petersilka, in Density Functional Theory II, Topics in Current Chemistry Vol. 181, edited by R. F. Nalewajski (Springer, Berlin, 1996).

[14] W. Kohn and L. J. Sham, Phys. Rev. 140, A1133 (1965).

[15] E. K. U. Gross and W. Kohn, Phys. Rev. Lett. 55, 2850 (1985).

[16] E. M. Lifshitz and L.P. Pitaevskii, Statistical Physics, in Course of Theoretical Physics Part II, Vol. 9, edited by L. D. Landau and E. M. Lifshitz (Reed Publishing, Oxford, 1980).

[17] The precise definition is $E[\mathbf{m}]=\min _{\psi \rightarrow \mathbf{m}(\mathbf{r})}\langle\psi|\hat{H}| \psi\rangle$, where $\hat{H}$ is the Hamiltonian and the minimum is sought among the wave functions that yield the magnetization $\mathbf{m}(\mathbf{r})$. This constrained search also yields the ground-state wave function $|\psi(\mathbf{m})\rangle$ as a functional of $\mathbf{m}(\mathbf{r})$.

[18] A general expression for the Berry curvature in terms of a linear response function is also derived in S. R. Jain and A. K. Pati, Phys. Rev. Lett. 80, 650 (1998).

[19] K. Burke, J. Perdew, and Y. Wang, in Electronic Density Functional Theory, Recent Progress and New Directions, edited by J. Dobson, G. Vignale, and M. P. Das (Plenum, New York, 1998), p. 81.

[20] Thus, we do not consider the possibility of spontaneous ferromagnetism in the homogeneous electron gas. This is predicted to occur at extremely low densities [21] and is of no interest here.

[21] D. M. Ceperley and B. J. Alder, Phys. Rev. Lett. 45, 566 (1980); G. Ortiz and P. Ballone, Phys. Rev. B 50, 1391 (1994); G. Ortiz, M. Harris, and P. Ballone, Phys. Rev. Lett. 82, 5317 (1999).

[22] B. I. Halperin and P.C. Hohenberg, Phys. Rev. 188, 898 (1969). Note that $\gamma_{i j}\left(\mathbf{r}, \mathbf{r}^{\prime}\right)$ in Eq. (17) is negative definite when $\gamma_{2, i j}>0$.

[23] R. Nifosì, S. Conti, and M. P. Tosi, Phys. Rev. B 58, 12758 (1998).

[24] P. Gori-Giorgi and J. P. Perdew, Phys. Rev. B 64, 155102 (2001). 